\title{
Urinary monocyte chemotactic protein 1 as a predictive marker of steroid responsiveness in children with idiopathic nephrotic syndrome A.S.El hamshary ${ }^{1}$, O.M.Abdel Haie ${ }^{1}$, W.E.Afifi ${ }^{1}$, A.A.El Falah ${ }^{2}$ and A.R.Mohammed \\ ${ }^{1}$ Pediatrics, Dept., Faculty of Medicine, Benha Univ., Benha, Egypt \\ ${ }^{2}$ Clinical and Chemical Pathology, Dept., Faculty of Medicine, Benha Univ., Benha, Egypt \\ E-mail: ashrafswidan2014@gmail.com
}

\begin{abstract}
Background: The role of pro-inflammatory cytokines in the immunopathogenesis of idiopathic nephrotic syndrome (INS) had been widely postulated. Reports on the release of cytokines, during idiopathic nephrotic syndrome activation, were conflicting in defining a specific interleukin pattern during relapse and remission of the disease. The monocyte chemoattractant protein-1 (MCP-1) is a member of the CC chemokine family, and a potent chemotactic factor for monocytes. It plays an important role in the recruitment of monocytes/macrophages into renal tubulointerstitium . Objective: The aim of this study was to determine the urinary level of monocyte chemotactic protein-1 (uMCP-1) in idiopathic nephrotic syndrome patients and its relationship to steroid sensitivity. Patients and Methods: This cross sectional study included 40 patients with nephrotic syndrome (NS) following up in Pediatric Nephrology Clinic, Benha University subdivided into 2 groups: group A (cases in remission) \& group B (cases in activity). Also, 20 age and sex matched healthy children have been included as a control group. Results: We found a highly significant difference in urinary MCP-1 between group A, group B, and controls, the highest being group B. The levels in patients in remission still exceeded the controls significantly. No difference was found between males and females in group A as regards urinary MCP-1 levels yet females had significantly higher levels in group B. This difference was not seen in controls. Conclusions: Urinary MCP-1 is highly sensitive and specific biomarker in idiopathic nephrotic syndrome.
\end{abstract}

Key words: Idiopathic Nephrotic Syndrome, Urinary Monocyte Chemotactic Protein-1, Relapse, Remission.

\section{Introduction}

Nephrotic syndrome is one of the most common glomerular disorders of childhood. It is characterized by proteinuria that is severe enough to cause hypoalbuminemia and edema [1].

The development of kidney disease involves a complex interplay between neurohormonal, inflammatory and biochemical changes which act on either intrinsic or extrinsic renal cells, or both. This can lead to the development of an innate immune response predominantly characterized by the accumulation and activation of leukocytes, particularly monocytes/ macrophages, in the kidney.

Chemokine-induced recruitment of peripheral leukocytes into tissues is a critical step in the development of inflammatory responses [2].

The role of pro-inflammatory cytokines in the immunopathogenesis of idiopathic nephrotic syndrome had been widely postulated. Reports on the release of cytokines, during idiopathic nephrotic syndrome (INS) activation, were conflicting in defining a specific interleukin pattern during relapse and remission of the disease [3].

The monocyte chemoattractant protein -1 (MCP-1) is a member of the $\mathrm{CC}$ chemokine family, and a potent chemotactic factor for monocytes [4]. It plays an important role in the recruitment of monocytes/macrophages into renal tubulointerstitium [5].

\section{Aim of the work}

The present study was designed to check urinary MCP-1 levels in pediatric patients with idiopathic nephrotic syndrome, and to detect effect of disease remission and activity on these levels and also to compare our results with the urinary levels of uMCP-1 in age and sex matched control group.

\section{Patients and Methods}

This study is a cross-sectional included fourty children with nephrotic syndrome; from those attending the pediatric nephrology clinic, Benha university hospital; and who were under regular follow up during the period from March 2020 to April 2021. Also twenty apparently healthy children age and sex matched as control group. The group of patients comprised 40 children; they were 17 males and 23 females. Their ages ranged from 4 years -14 years.

\subsection{Inclusion criteria}

included children and adolescents with wellestablished INS with still preserved renal function, followed-up from 2020 to 2021, whose parents gave their consent to participate in the study protocol.

\subsection{Exclusion criteria}

Patients with renal impairment (high serum creatinine level) or patients with any manifestations of systemic disease or patients with secondary nephrotic syndrome.

The patients were classified into 2 groups:

- Group A: This group included 20 nephrotic patients in remission. It included 8 males and 12 females, their ages ranged from 4.5 -14 years.

- Group B: This group included 20 nephrotic patients in activity. It included 9 males and 11 females, their ages ranged from 4.1 -13.1 years.

Control group: The control group comprised twenty (20) age and sex matched healthy children 8 
males and 12 females, their ages ranged from 5.5 -12 years.

Ethical aspects: The Ethics Committee of the Benha Univeristy Faculty of Medicine Pediatric Department approved the study. Informed consent was obtained from parents of all included patients. The research protocol did not interfere with any medical recommendations or prescriptions.

\section{All children will be subjected to the following:}

1. Full history taking.

2. Clinical examination including: a) General examination b) Local examination examination.

3. Investigations: a) Routine lab investigations: Including complete blood count, C-reactive protein , serum creatinine, blood urea, serum albumin, serum cholesterol, complete urine analysis, and urine protein/ creatinine ratio. Quantitative determination of urinary monocyte chemotactic protein-1 (uMCP-1) using ELISA technique.

Immunoassays of uMCP-1 cytokine. Freshly voided urine samples were collected by sterile tube and centrifuged at 2000 RPM for 20 minutes. The supernatant was then collected, aliquoted and immediately frozen at $-80 \mathrm{C}$ till the time of assessment.

\subsection{Statistical analysis}

Data entry, processing and statistical analysis was carried out using Statistical package for social sciences (IBM-SPSS), version 24 (May 2016); IBM- Chicago, USA will be used for statistical data analysis. Tests of significance (Kruskal-Wallis, Wilcoxon's, Chi square, logistic regression analysis, and Spearman's correlation) were used. Data were presented and suitable analysis was done according to the type of data (parametric and nonparametric) obtained for each variable. P-values less than $0.05(5 \%)$ was considered to be statistically significant.

\section{Results}

Table (1) shows that group B (cases in activity) had the lowest level of the serum albumin followed by group A (cases in remission) then control group, the differences between the three groups were statistically highly significant. In contrast, group B (cases in activity) had the highest level of serum cholesterol and protein/creatinine ratio followed by group A (cases in remission) then control group, again, the differences between the three groups were statistically highly significant.

Table (2) shows that the mean value of uMCP-1 was significantly higher among cases than control group and the difference was statistically highly significant.

\section{Discussion}

Nephrotic syndrome in children is defined as hypoalbuminemia, edema, and proteinuria (protein > $40 \mathrm{mg} / \mathrm{m}^{2} / \mathrm{h}$ or protein-creatinine ratio $>2000 \mathrm{mg} / \mathrm{g}$ (>200 $\mathrm{mg} / \mathrm{mmol}$ ] or protein > $300 \mathrm{mg} / \mathrm{dL}$ or $3+$ on urine dipstick [in adults, proteinuria > $3.5 \mathrm{~g}$ per 24 hours]) [6].

Although the pathogenesis and pathophysiology of idiopathic NS is not clearly understood, it is reported that the immune system, including activation of lymphocytes, plays an important role. An association among several cytokines and chemokines with idiopathic NS has been reported [7].

Monocyte chemoattractant protein-1(MCP-1) belongs to the CC-chemokine family, is encoded on chromosome 17, and is composed of 76 amino acids. In the kidney, it is produced by mesangial, tubular, epithelial cells, and in smooth muscle. It is mainly expressed by monocytes, activated macrophages, $\mathrm{T}$ cells, and NK cells [8].

MCP-1 triggers migration and retention of monocytes and transformation of fibroblasts in the glomeruli, playing an important role in the glomerular inflammation [9].

Our study has female predominance about 54\% and that is in agreement with Agrawal et al., [10] study in which his study group has also female predominance that represent $55 \%$.

Table (1) Comparison between group A (cases in remission), group B (cases in activity ) \& control group regarding serum albumin, cholesterol and protein/ creatinine ratio.

\begin{tabular}{lccccc}
\hline & Group A (20) & Group B (20) & \multicolumn{2}{c}{ Control (20) } & P. value \\
& Mean \pm SD & Mean \pm SD & Mean \pm SD & \\
\hline S. Albumin (g/dl) & $3.07 \pm 0.224$ & $2.1 \pm 0.41$ & $3.5 \pm 0.33$ & $<0.001$ \\
Cholesterol (mg/dl) & $157.7 \pm 10.71$ & $275.1 \pm 47.87$ & $91.35 \pm 8.57$ & $<0.001$ \\
Protein/creatinine ratio (mg/g Cr) & $0.995 \pm 0.265$ & $19.67 \pm 6.25$ & $0.07 \pm 0.03$ & $<0.001$ \\
\hline
\end{tabular}

Table (2) Comparison between cases and control group regarding uMCP-1.

\begin{tabular}{lcll}
\hline & Cases (40) & Control (20) & P. value \\
& Mean \pm SD & Mean \pm SD & \\
\hline uMCP-1 (pg/dl) & $231.6 \pm 177.9$ & $47.2 \pm 15.3$ & $<0.001$ \\
\hline
\end{tabular}


Our study showed no significant difference was found between patients \& control group regarding blood pressure. This is in agreement with the study done by Filha et al., [11] that found no significant difference between INS patients and control group as regard blood pressure. Arterial hypertension is commonly encountered by clinicians treating children with steroid sensitive and steroid resistant nephrotic syndrome. Multiple mechanisms and co-morbidities contribute to the pathophysiology of hypertension in children with NS. Some contributing factors are known to cause acute and episodic elevations in blood pressure such as fluid shifts, sodium retention, and medication side effects (steroids, calcineurin inhibitors). Others are associated with chronic and more sustained hypertension such as renal fibrosis, decreased GFR, and progression of chronic kidney disease. Children with NS are more likely to suffer from other cardiovascular disease risk factors, such as obesity, increased measures of arterial stiffness [increased carotid intima-media thickness, endothelial dysfunction, increased pulse wave velocity], impaired glucose metabolism, dyslipidemia, left ventricular hypertrophy, left ventricular dysfunction, and atherosclerosis [12].

In our study, there was highly statistical significant difference between patients and control group regarding presence of edema $(\mathrm{p}<0.001)$. On other hand, $76 \%$ of patients in activity (group B) were presented by facial \& L.L edema, and no one of patients in remission (group A) or in control group presented by edema. Ejaz et al., [13] study reported that $65 \%$ of cases in active nephrotic syndrome presented by facial \& L.L. edema which agrees with our results.

In our study, there was no statistically significant difference between patients and control group as regard $\mathrm{CBC}$ and CRP.

Our study was consistent with the study done by Vianna et al., [14] that revealed no significant difference between patients and control group as regard CBC and CRP.

In our study, there was no statistically significant difference between patients and control group as regard serum creatinine and blood urea.

Our study agrees with Souto et al., [15] who reported that no differences were detected, in nitrogen waste levels (serum creatinine and blood urea), between INS groups and controls.

Our study observed highly statistical significant difference in uMCP-1 levels between the patients and control group ( $\mathrm{p}<0.001)$.

Also, this study was in agreement with the study done by Wasilewska et al., [16] that showed uMCP-1 in children with minimal change disease in relapse (IA) was significantly higher than in group IB (MCD after proteinuria regression) and controls ( $\mathrm{p}<0.05)$.

Moreover, our study is in agreement with Angela [17] study that reported a statistically increase in the level of urinary MCP-1 in all groups of patients (SRNS, SSNS), compared to the control group. The concentration of urinary MCP-1 in the groups of patients with SSNS and SRNS, during the clinical manifestations and remission period exceeded $2.0-2.4$ times the values of the control group which is statistically significant.

Our study showed highly statistical significant difference between SRNS and SSNS as regard uMCP-1 in all studied patients $(\mathrm{p}<0.001)$.

Angela [17] noted that the group of patients with SRNS showed higher mean values of MCP-1 in the urine compared to those in the group with SSNS ( $\mathrm{p}<$ 0.001).

\section{Conclusion}

Urinary MCP-1 highly sensitive and specific biomarker in childhood nephrotic syndrome for early detection of disease activity as its level is markedly elevated early in the course of the disease.

\section{References}

[1] A. Pan, D. Jana, "Relation of Steroid on Linear Growth Velocity with Biochemical Bone Markers in Children with Nephrotic Syndrome in a Teaching Hospital, Central Nepal," J Med Sci.Nepal; vol. 16(1), pp. 49-53,2020.

[2] M. J. Kim, F. W. Tam, "Urinary monocyte chemoattractant protein-1 in renal disease," Clinica chimica acta; vol. 412(24), pp. 2022-2030, 2011.

[3] A. A. Al-Eisa, M. Al Rushood, R. J. Al-Attiyah, "Urinary excretion of IL-1 $\beta$, IL-6 and IL-8 cytokines during relapse and remission of idiopathic nephrotic syndrome," J Inflam Res; vol.10(3),pp.1-12,2017.

[4] S. L. Deshmane, S. Kremlev, S. Amini, B. E. Sawaya, "Monocyte chemoattractant protein-1 (MCP-1): an overview, J Interf Cyto Res; vol.29(6),pp. 313-32,2009.

[5] T. Wada, H. Yokoyama, K. Matsushima, K. I. Kobayashi, "Monocyte chemoattractant protein-1: does it play a role in diabetic nephropathy?, "Nephrol Dialysis Transplan; vol.18(3),pp. 457-459,2003.

[6] K. Hampson, M. L. Gay, M. E. Band, "Pediatric Nephrotic Syndrome: Pharmacologic and Nutrition Management," Nutri Clin Prac; vol.36(2),pp.331-343,2021.

[7] Y. Matsumoto, Y. Ikezumi, T. Kondo,Y. Nakajima, Y. Yamamoto, M. Morooka, T. Yoshikawa, "Urinary monocyte chemotactic protein 1 as a predictive marker of steroid responsiveness in children with idiopathic nephrotic syndrome, Fujita Med J; vol. 4(1),pp.17-22,2018.

[8] C. C. Aragón, R. A. Tafúr, A. Suárez-Avellaneda, M. T. Martínez, A. de Las Salas, G. J. Tobón, "Urinary biomarkers in lupus nephritis," J Transl Autoimmun; vol. 3(12), pp.1042-1051,2020.

[9] S. Mao, L. Wu, "Association between MCP-1 $2518 \mathrm{~A}>\mathrm{G}$ gene olymorphism and chronic 
kidney disease," Internat Urol Nephrol; vol. 50(12), pp.2245-2253,2018.

[10] S. Agrawal, M. E. Brier, B. A. Kerlin, W. E. Smoyer, J. Mahan, H. Patel, L. A. Greenbaum, "Plasma Cytokine Profiling to Predict Steroid Resistance in Pediatric Nephrotic Syndrome," Kidn Internat Rep; vol. 6(3), pp.785795,2021.

[11]R. D. S. Filha, S. V. B. Pinheiro, T. Macedo e Cordeiro, V. Feracin, É. L. M. Vieira, A. S. Miranda, A. C. Simões e Silva, "Evidence for a role of angiotensin converting enzyme 2 in proteinuria of idiopathic nephrotic syndrome," Biosci. Rep; vol. 39(1),pp. 113,2019 .

[12]I. F. Shatat, L. J. Becton, R. P. Woroniecki, "Hypertension in childhood nephrotic syndrome," Frontiers in Pediatr; vol 7(4),pp. 287 $296,2019$.

[13] I. Ejaz, H. I. Khan, B. K. Javaid, G. Rasool, M. T. Bhatti, "Histopathological diagnosis and outcome of paediatric nephrotic syndrome," J the College Physicians Surgeons--pakistan: JCPSP; vol. 14(4),pp. 229-233,2001.

[14]H. R. Vianna, C. Soares, K. D. Silveira, G. S. Elmiro, P. M. Mendes, M. de Sousa Tavares, A. C. S. Silva, "Cytokines in chronic kidney disease: potential link of MCP-1 and dyslipidemia in glomerular diseases," Pedia Nephrol; vol. 28(3), pp.463-469,2013.

[15]M. F. Souto, A. L.Teixeira, R. C. Russo, M. Penido, K. D. Silveira, M. Teixeira, A. Silva, "Immune mediators in idiopathic nephrotic syndrome: evidence for a relation between interleukin 8 and proteinuria," Pediatr Res; vol. 64(6),pp. 637-642,2008.

[16]A. Wasilewska, W. Zoch-Zwierz, K. TarantaJanusz, Z. Kołodziejczyk, "Urinary monocyte chemoattractant protein-1 excretion in children with glomerular proteinuria," Scandinavian J urol and Nephrol; vol.45(1), pp.52-59,2011.

[17]C. Angela, "The role of cell signaling molecules in the pathogenesis of glomerulonephritis in children," The Moldovan Med J; vol. 64(2), pp.37-41,2021. 